\title{
A Statistical Study on Force-Freeness of Solar Magnetic Fields in the Photosphere
}

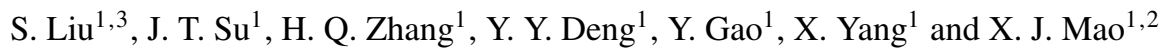 \\ ${ }^{1}$ National Astronomical Observatory and Key Laboratory of Solar Activity, Chinese Academy of Sciences, Beijing 100012, China \\ ${ }^{2}$ Department of Astronomy, Beijing Normal University, Beijing 100875, China \\ ${ }^{3}$ Email: lius@nao.cas.cn
}

(Received April 13, 2012; Accepted June 28, 2012; Online Publication January 24, 2013)

\begin{abstract}
It is an indisputable fact that solar magnetic fields are force-free in the corona, where force-free fields mean that current and magnetic fields are parallel and there is no Lorentz force in the fields. While the force-free extent of photospheric magnetic fields remains open, in this paper, we give its statistical results. Vector magnetograms (namely, Bx, By, and Bz in heliocentric coordinates) employed are observed by the Solar Magnetic Field Telescope at Huairou Solar Observing Station. We study and calibrate 925 magnetograms calibrated by two sets of calibration coefficients, which indicate the relation between magnetic fields and the strength of the Stokes spectrum and can be calculated either theoretically or empirically. The statistical results show that the majority of active region magnetic fields are not consistent with the force-free model.
\end{abstract}

Keywords: Sun: magnetic fields, Sun: corona, Sun: photosphere

\section{INTRODUCTION}

Magnetic fields dominate most solar activities such as filaments eruption, flares, and coronal mass ejections. All these phenomena are energetic events due to explosive release of magnetic energy (Krall et al. 1982; Wang, Zhang \& Xu 1994; Shibata et al. 1995; Tsuneta 1996; Bond et al. 2001; Priest \& Forbes 2002; Lin et al. 2005; Nindos, Patsourakos \& Wiegelmann 2012). Thus, the solar magnetic field is the key for understanding the nature of solar activities. Since measurable magnetic sensitive lines are around the photosphere, reliable measurements of magnetic fields are nearly there (Stenflo 1973; Harvey 1977). However, the understanding of the magnetic field in the chromosphere and corona remains difficult due to both intrinsic physical difficulties and observational limitations (Gary \& Hagyard 1990; Gary \& Hurford 1994; Liu, Kuhn \& Coulter 2004). Generally, magnetic fields in the corona are regarded as force-free (Aly 1989), because the plasma $\beta$ (ratio of plasma pressure to magnetic pressure) is much less than unity. However, it is controversial in the photosphere, because two kinds of pressures are comparable (Demoulin et al. 1997). For the low- $\beta$ corona where the plasma is tenuous $(\beta \ll 1)$, the magnetic field satisfies the following force-free equations:

$$
\nabla \times \boldsymbol{B}=\alpha(\boldsymbol{r}) \boldsymbol{B}
$$

$$
\nabla \cdot \boldsymbol{B}=0
$$

implying that there is no Lorentz force in action and $\alpha$ is constant along magnetic field lines $(\boldsymbol{B} \cdot \nabla \alpha=0)$.

At present, magnetic field extrapolation with a force-free assumption is a major method for studying the solar magnetic fields of active regions. Coronal fields can be reconstructed from a physical model (namely, the force-free model) in which the observed photospheric magnetic field is taken as a boundary condition (Sakurai 1981; Wu et al. 1990; Mikic \& McClymont 1994; Amari et al. 1997; Wheatland, Sturrock \& Roumeliotis 2000; Yan \& Sakurai 2000; Wiegelmann 2004; Song et al. 2006; He \& Wang 2008; Liu, Zhang \& Su 2011a). This means that coronal magnetic fields are considered to be force-free, while at the boundary these are connected to the photospheric magnetic fields observed. The forcefree extent of the photospheric magnetic field then becomes an important subject to study. Wiegelmann, Inhester, \& Sakurai (2006) proposed a preprocessing procedure to make a minor regulation within the allowable errors, so that the observed magnetic fields tend to a force-free field, which further indicates that the study of the force-free extent is significant and necessary for the field extrapolation. Metcalf et al. (1995) calculated the dependence of the net Lorentz force in the photosphere and low chromosphere on the height using Mees Solar Observatory magnetograms and concluded 
that magnetic fields are not force-free in the photosphere, but become force-free roughly $400 \mathrm{~km}$ above the photosphere. Moon et al. (2002), studying the force-free extent in the photosphere using 12 vector magnetograms of three active regions, realised that the photospheric magnetic fields are not very far from the force-free case. Liu et al. (2011b) tentatively applied the force-free extrapolation to reconstruct the magnetic fields above the quiet region and checked the force-free extent of this quiet region based on the high spatial resolution vector magnetograms observed by the Solar Optical Telescope/Spectro-Polarimeter on board Hinode. Tiwari (2012) found that sunspot magnetic fields are not so far from the force-free case. We conduct statistical research to make use of the vector magnetograms observed by the SMFT at HSOS from 1988 to 2001 in order to verify the force-freeness of the photospheric magnetic field.

This paper is organised as follows. The description of observations and data reduction is given in Section 2. The results are shown in Section 3. Finally, in Section 4, we present short discussions and conclusions.

\section{OBSERVATIONS AND DATA REDUCTION}

The observational data used here are 925 vector magnetograms corresponding to 925 active regions observed from 1988 to 2001 by the Solar Magnetic Field Telescope (SMFT) installed at Huairou Solar Observing Station (HSOS), located at the north shore of the Huairou reservoir. Magnetograms associated with the corresponding active region, which is nearest to the disk center, are chosen and calculated. Therefore, only one magnetogram is available for one active region. The SMFT consists of a $35-\mathrm{cm}$ refractor with a vacuum tube, a birefringent filter, and a CCD camera, including an image processing system operated by a computer (Ai \& Hu 1986). The birefringent filter is tunable, working either at the photosphere line Fe I $\lambda 5324.19 \AA$, with a $0.150-\AA$ bandpass, or at the chromosphere line, $\mathrm{H} \beta$, with a $0.125-\AA$ bandpass. The line of Fe I $\lambda 5324.19 \AA$ (Lande factor $g=1.5$ ), formed around the solar photosphere, is used for photospheric magnetic field observations. The bandpass of the birefringent filter is about $0.15 \AA$ for the Fe I $\lambda 5324.19 \AA$ line. The center wavelength of the filter can normally be shifted $-0.075 \AA$ relative to the center of $\mathrm{Fe}$ I $\lambda 5324.19 \AA$ to measure the longitudinal magnetic field and then the line center is applied to measure the transverse one (Ai \& Hu 1986). Vector magnetograms are reconstructed from four narrow-band images of Stokes parameters $(I, Q, U$, and $V)$. Note that $V$ is the difference of the left and right circularly polarised images, $Q$ and $U$ are the differences between two orthogonal linearly polarised images for different azimuthal directions, $I$ is the intensity derived from the sum either of two circularly polarised images in the line-of-sight field measurements or of two linearly polarised images in the transverse field measurement. When $I, Q, U$, and $V$ are measured, the corresponding white light images are simultaneously obtained, which are employed to compensate for the time differences during the measurements of $I, Q, U$, and $V$. The sequence of obtaining Stokes images is as follows. First, acquire the $V / I$ image, next the $Q / I$ images, and then the $U / I$ image. The time required to obtain a set of Stokes images was about $45 \mathrm{~s}$. Each image is associated with 256 integrated frames. To reconstruct the vector magnetograms, a linear relation is necessary between the magnetic field and the Stokes parameters $I, Q, U$, and $V$, which is true under the weak-field approximation (Jefferies, Lites \& Skumanich 1989; Jefferies \& Mickey 1991):

$$
\begin{gathered}
B_{\mathrm{L}}=C_{\mathrm{L}} V, \\
B_{\mathrm{T}}=C_{\mathrm{T}}\left(Q^{2}+U^{2}\right)^{1 / 4}, \\
\theta=\arctan \left(\frac{B_{\mathrm{L}}}{B_{\perp}}\right), \\
\phi=\frac{1}{2} \arctan \left(\frac{U}{Q}\right),
\end{gathered}
$$

where $B_{\mathrm{L}}$ and $B_{\mathrm{T}}$ are the line-of-sight and transverse components of the photospheric field, respectively. Here, $\theta$ is the inclination between the vector magnetic field and the direction normal to the solar surface and $\phi$ is the field azimuth. Furthermore, $C_{\mathrm{L}}$ and $C_{\mathrm{T}}$ are the calibration coefficients for the longitudinal and transverse magnetic fields, respectively. Both theoretical and empirical methods are used to calibrate vector magnetograms (Wang, Ai, \& Deng 1996; Ai, Li \& Zhang 1982), so that two sets of calibration coefficients are available: the first set $C_{\mathrm{L}}$ and $C_{\mathrm{T}}$ are 8381 and $6790 \mathrm{G}$ (Su \& Zhang 2004; Wang et al. 1996), respectively, obtained by theoretical calibration; in the second set $C_{\mathrm{L}}$ and $C_{\mathrm{T}}$ are 10000 and $9730 \mathrm{G}$, respectively, which are deduced through empirical calibrations (Wang et al. 1996). Faraday rotation and magneto-optical effects may affect the value of measured magnetic fields. Bao et al. (2000) analyzed the Faraday rotation in the HSOS magnetograms, which contributes about $12^{\circ}$. Gao et al. (2008) proposed a way of the statistical removal of Faraday rotation in vector magnetograms from HSOS. Zhang (2000) found that the statistical mean azimuth error of the transverse field is $12.8^{\circ}$ caused by magnetooptical effects. In addition, there may be other causes for the data uncertainty, for example saturation effects, filling factors, and stray light. After routine data processing of HSOS data, the spatial resolution of observational data is actually $2 \times 2\left(\operatorname{arcsec} \text { pixel }^{-1}\right)^{2}$ and $3 \sigma$ noise levels of vector magnetograms are 20 and $150 \mathrm{G}$ for longitudinal and transverse components, respectively.

The acute angle method is employed to resolve the $180^{\circ}$ ambiguity (Wang, Zhang, \& Ai 1994; Wang 1997; Wang, Yan, \& Sakurai 2001; Metcalf et al. 2006), in which the observed field is compared with the extrapolated potential field in the photosphere. The orientation of the observed transverse component is chosen by requiring $-90^{\circ} \leq \Delta \theta \leq 90^{\circ}$, where $\Delta \theta=\theta_{\mathrm{o}}-\theta_{\mathrm{e}}$ is the angle difference between the observed and extrapolated transverse components. To minimise the projection effects, the requirement that the horizontal width 

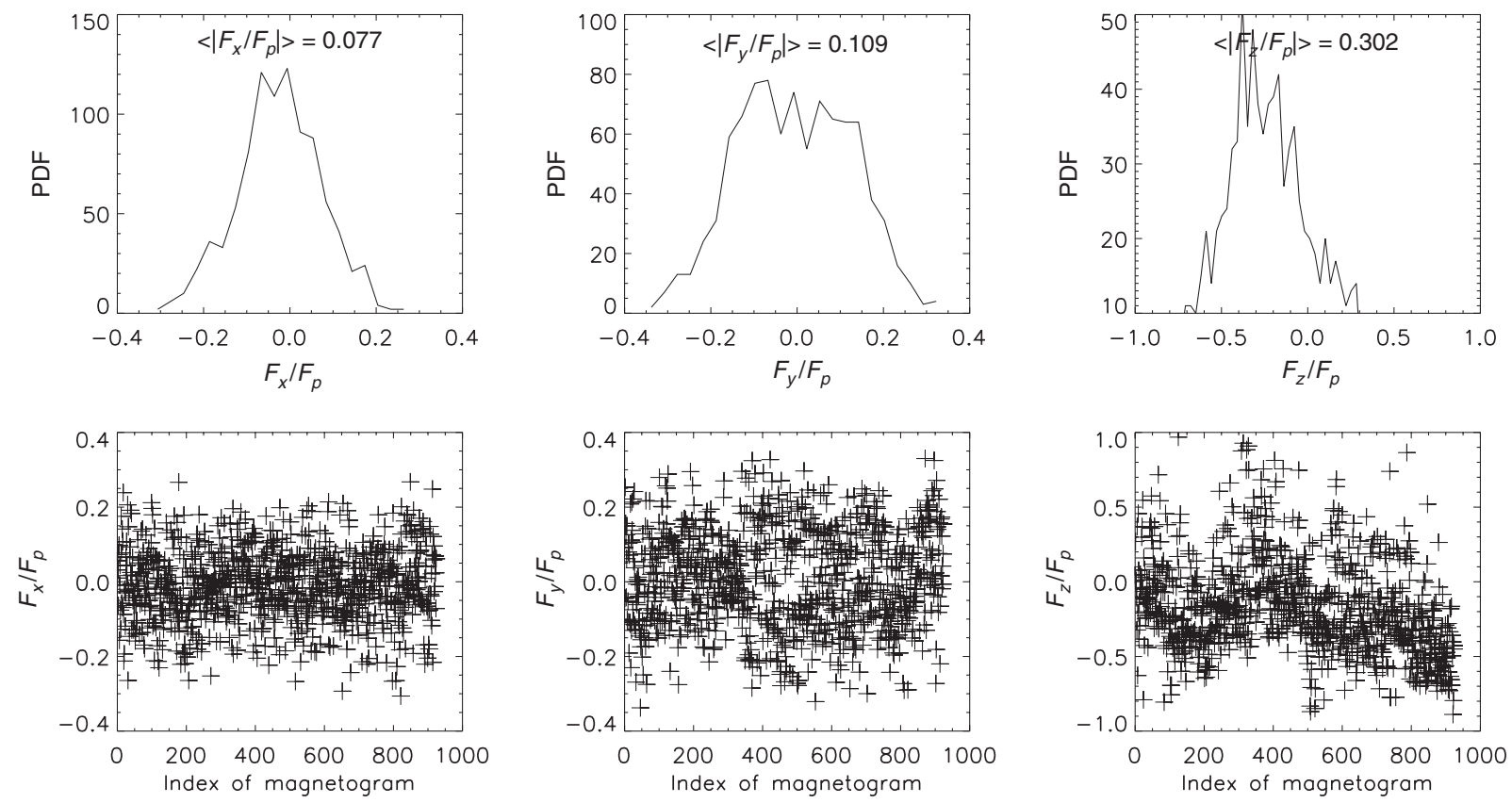

Figure 1. PDF and scatter diagrams of $F_{x} / F_{p}, F_{y} / F_{p}$, and $F_{z} / F_{p}$ for the selected magnetograms. Mean values of absolute $F_{x} / F_{p}, F_{y} / F_{p}$, and $F_{z} / F_{p}$ are plotted and indicated by $\left\langle\left|F_{x} / F_{p}\right|>,<\left|F_{y} / F_{p}\right|>\right.$, and $\left\langle\left|F_{z} / F_{p}\right|>\right.$, respectively (Case I).

of an active region is less than $20^{\circ}$ is added for each magnetogram.

\section{RESULTS}

In classical electromagnetic theory, the Lorentz force can be written as the divergence of the Maxwell stress:

$$
\boldsymbol{F}=\frac{(\boldsymbol{B} \cdot \boldsymbol{\nabla}) \boldsymbol{B}}{4 \pi}-\frac{\nabla(\boldsymbol{B} \cdot \boldsymbol{B})}{8 \pi} .
$$

Assuming that the magnetic field above the plane $z=0$ (namely, the photosphere) falls off fast enough as going to infinity, the net Lorentz force in the infinite half-space $z>$ 0 is just the Maxwell stress integrated over the plane $z=0$ (Chandrasekhar 1961; Molodensky 1974; Aly 1984; Low 1985). Then, the components of the net Lorentz force at the plane $\mathrm{z}=0$ can be expressed by the surface integrals as follows:

$$
\begin{gathered}
F_{x}=-\frac{1}{4 \pi} \int B_{x} B_{z} \mathrm{~d} x \mathrm{~d} y, \\
F_{y}=-\frac{1}{4 \pi} \int B_{y} B_{z} \mathrm{~d} x \mathrm{~d} y, \\
F_{z}=-\frac{1}{8 \pi} \int\left(B_{z}^{2}-B_{x}^{2}-B_{y}^{2}\right) \mathrm{d} x \mathrm{~d} y .
\end{gathered}
$$

The necessary conditions of a force-free field are that all three components are much less than $F_{p}$ (Low 1985), where $F_{p}$ is a characteristic magnitude of the total Lorentz force and can be written as

$$
F_{p}=\frac{1}{8 \pi} \int\left(B_{z}^{2}+B_{x}^{2}+B_{y}^{2}\right) \mathrm{d} x \mathrm{~d} y .
$$

The values of $F_{x} / F_{p}, F_{y} / F_{p}$, and $F_{z} / F_{p}$ then provide a measure of the force-free extent at the boundary plane $z=0$ (the photosphere).

Following Metcalf et al. (1995) and Moon et al. (2002), $F_{x} / F_{p}, F_{y} / F_{p}$, and $F_{z} / F_{p}$ are utilised to check the force-free extent of the selected photospheric magnetograms. The necessary conditions of the force-free field are also expressed as $\left|F_{x}\right| / F_{p} \ll 1,\left|F_{y}\right| / F_{p} \ll 1$, and $\left|F_{z}\right| / F_{p} \ll 1$ (Metcalf et al. 1995; Moon et al. 2002), that is if three parameters $F_{x} / F_{p}$, $F_{y} / F_{p}$, and $F_{z} / F_{p}$ are so small that they are negligible, then the magnetic field can be regarded as force-free completely. Metcalf et al. (1995) suggested that the magnetic field is force-free if $F_{z} / F_{p}$ is less than or equal to 0.1 . The calibration coefficients $C_{\mathrm{L}}$ and $C_{\mathrm{T}}$ may affect the three parameters of $F_{x} / F_{p}, F_{y} / F_{p}$, and $F_{z} / F_{p}$. Thus, two sets of calibration coefficients mentioned above are applied to this study. In Case I, $C_{\mathrm{L}}$ and $C_{\mathrm{T}}$ are chosen as 8381 and $6790 \mathrm{G}$ (Wang et al. 1996; Su \& Zhang 2004), respectively. In Case II, $C_{\mathrm{L}}$ and $C_{\mathrm{T}}$ are chosen as 10000 and $9730 \mathrm{G}$ (Wang et al. 1996), respectively.

Figure 1 shows the possibility density function (PDF) and scatter diagrams of $F_{x} / F_{p}, F_{y} / F_{p}$, and $F_{z} / F_{p}$ for the selected magnetograms (Case I). The mean values of absolute $F_{x} / F_{p}, F_{y} / F_{p}$, and $F_{z} / F_{p}$ for all selected 925 magnetograms 

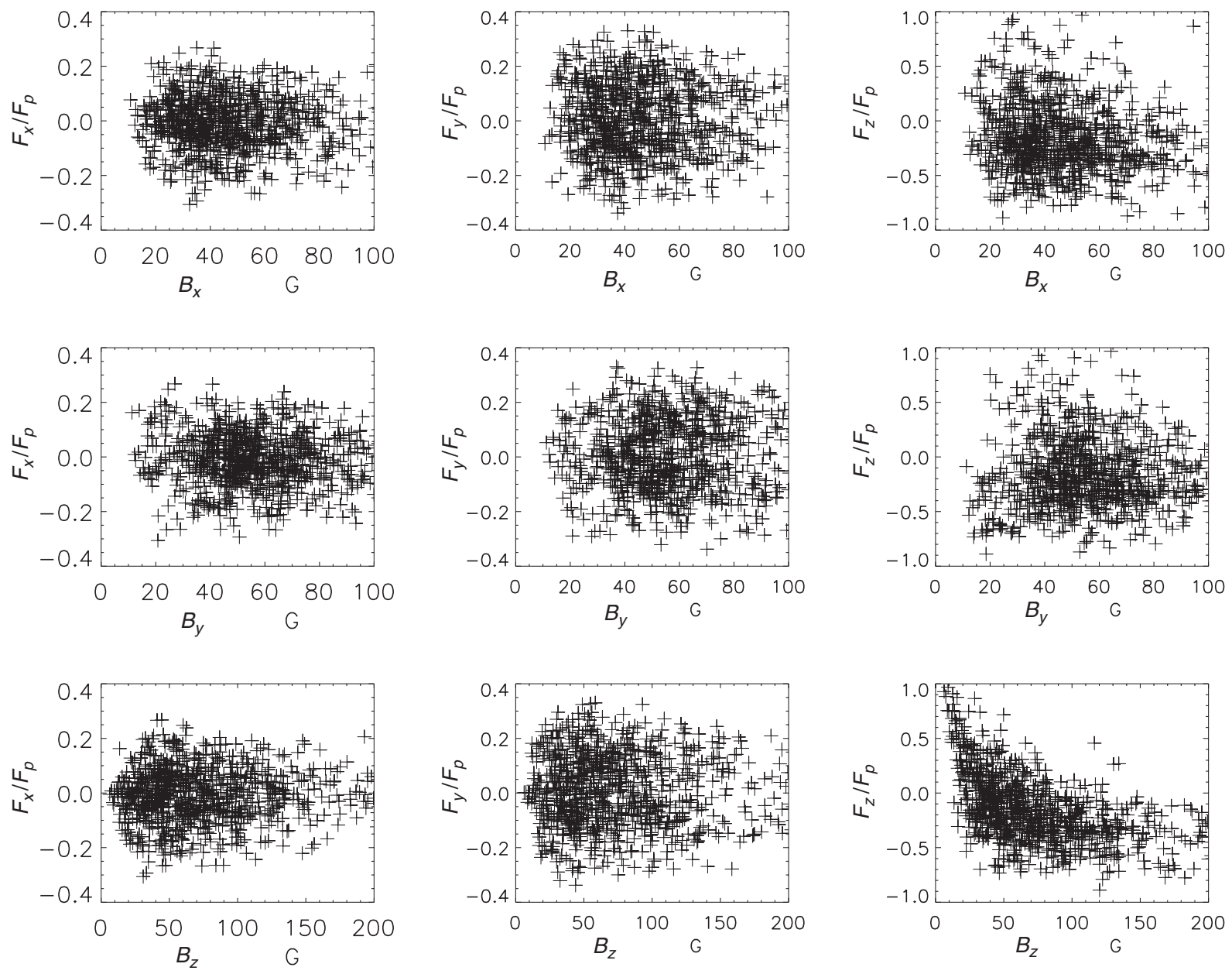

Figure 2. $F_{x} / F_{p}, F_{y} / F_{p}$, and $F_{z} / F_{p}$ vs. $B_{x}, B_{y}$, and $B_{z}$ for the selected magnetograms (Case I).

are $0.077,0.109$, and 0.302 , respectively. The amplitudes of $F_{x} / F_{p}$ and $F_{y} / F_{p}$ are evidently smaller than that of $F_{z} / F_{p}$, which is the same as in the previous study (Metcalf et al. 1995). Therefore, $F_{z} / F_{p}$ can work as a criterion indicating a force-free or non-force-free field more evidently. From the distribution of PDF and scatter diagrams, most of the magnetograms have the amplitudes of $F_{z} / F_{p}$ distributed outside the zone consisting the width of \pm 0.1 , which means that most of the photospheric magnetic fields deviate from a forcefree field. There are about $17 \%$ of the magnetograms with values of $F_{z} / F_{p}$ less than 0.1 for Case I (38\% of the magnetograms with values of $F_{z} / F_{p}$ less than 0.2$)$. To see the relation between $F_{x} / F_{p}, F_{y} / F_{p}$, and $F_{z} / F_{p}$ and the magnetic field strength, Figure 2 shows $F_{x} / F_{p}, F_{y} / F_{p}$, and $F_{z} / F_{p}$ versus the magnetic components of $B_{x}, B_{y}$, and $B_{z}$ for the selected magnetograms (Case I), where $B_{x}, B_{y}$, and $B_{z}$ are the average of the absolute values of all pixels for each magnetogram. It can be seen only at the bottom right of Figure 2 that the amplitudes of $F_{z} / F_{p}$ decrease roughly as $B_{z}$ increases, and no evident correlation exists between the parameters $\left(F_{x} / F_{p}\right.$, $F_{y} / F_{p}$, or $\left.F_{z} / F_{p}\right)$ and magnetic components, but according to Equation (10), the value of $F_{z} / F_{p}$ should decrease as $B_{z}$ increases.

To study the effect of calibration coefficients on these three parameters of $F_{x} / F_{p}, F_{y} / F_{p}$, and $F_{z} / F_{p}$, PDF and scatter diagrams of $F_{x} / F_{p}, F_{y} / F_{p}$, and $F_{z} / F_{p}$ of the selected magnetograms (Case II) are plotted in Figure 3. Like case I, the amplitudes of $F_{x} / F_{p}$ and $F_{y} / F_{p}$ are also smaller than that of $F_{z} / F_{p}$ in case II. For Case II, the mean values of absolute $F_{x} / F_{p}, F_{y} / F_{p}$, and $F_{z} / F_{p}$ for all selected magnetograms are $0.078,0.111$, and 0.251 , respectively, which are smaller than those of Case I on the whole. Moreover, for Case II there are about $25 \%$ of the magnetograms with the value of $F_{z} / F_{p}$ less than 0.1 (49\% of the magnetograms with the values of $F_{z} / F_{p}$ less than 0.2). It should be noted that though more $F_{z} / F_{p}$ in the zone mentioned above (consisting a width of \pm 0.1 ), most of the magnetograms cannot be regarded as force-free. Besides, the widths of PDF are not narrow and the scatter of diagrams is diverge as well. In general, there is a deviation of $F_{z} / F_{p}$ from the zone. For Case II, $F_{x} / F_{p}, F_{y} / F_{p}$, and $F_{z} / F_{p}$ versus magnetic components of $B_{x}, B_{y}$, and $B_{z}$ are plotted in Figure 4 to study their relations with the magnetic field 

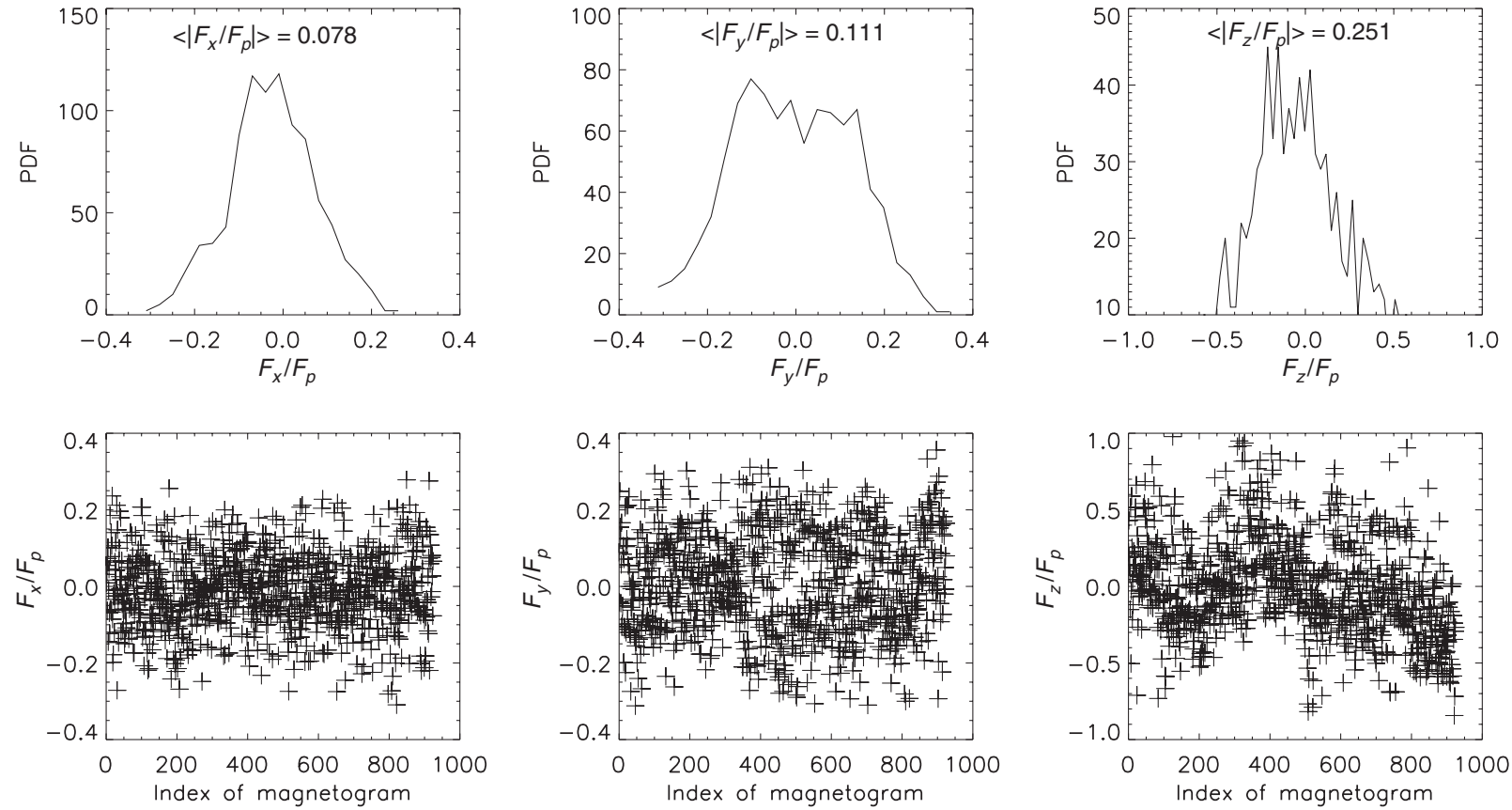

Figure 3. PDF and scatter diagrams of $F_{x} / F_{p}, F_{y} / F_{p}$, and $F_{z} / F_{p}$ for the selected magnetograms. Mean values of absolute $F_{x} / F_{p}, F_{y} / F_{p}$, and $F_{z} / F_{p}$ are plotted and indicated by $\left\langle\left|F_{x} / F_{p}\right|>,\left\langle\left|F_{y} / F_{p}\right|>\right.\right.$, and $<\left|F_{z} / F_{p}\right|>$, respectively (Case II).
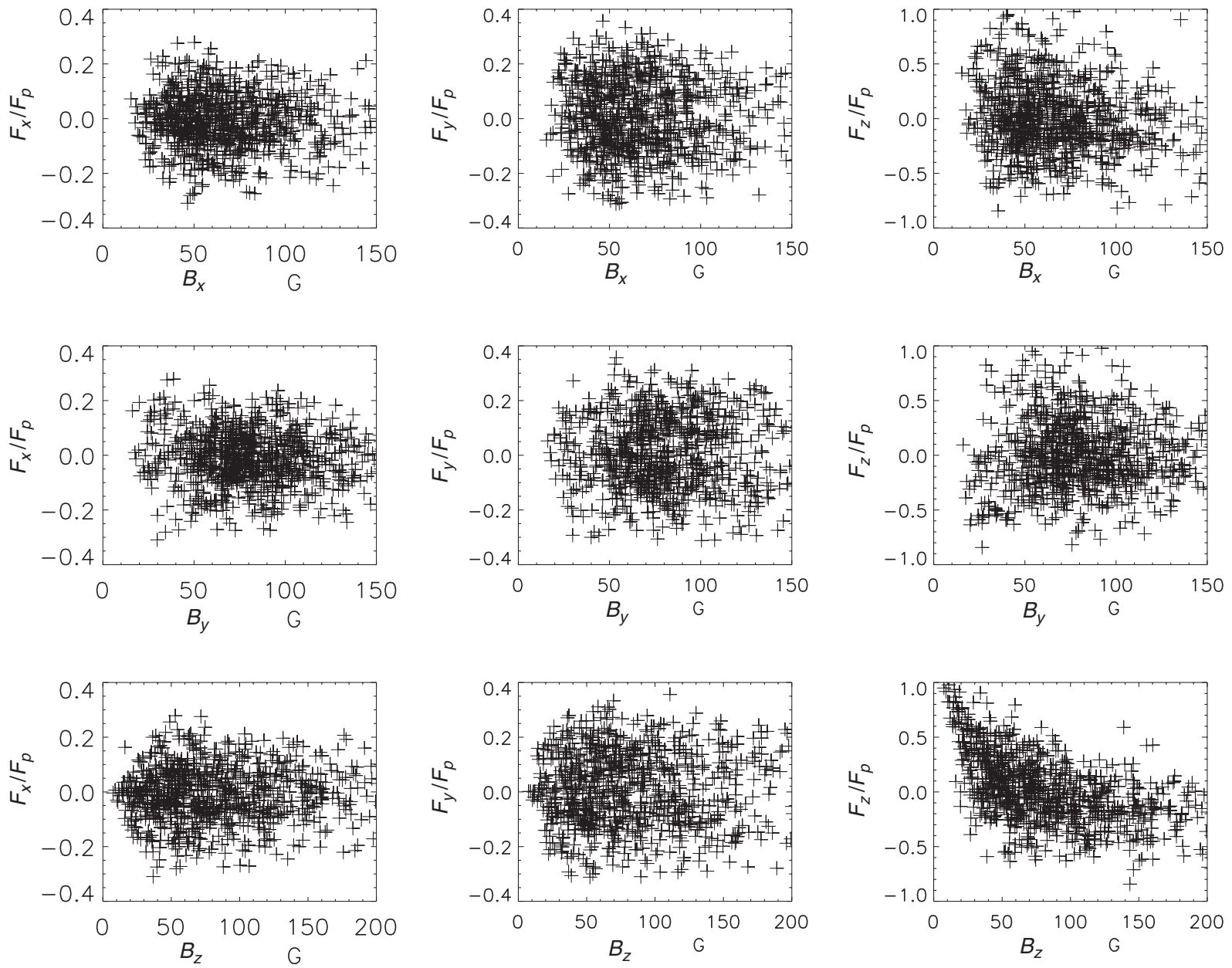

Figure 4. $F_{x} / F_{p}, F_{y} / F_{p}$, and $F_{z} / F_{p}$ vs $B_{x}, B_{y}$, and $B_{z}$ for the selected magnetograms (Case II). 

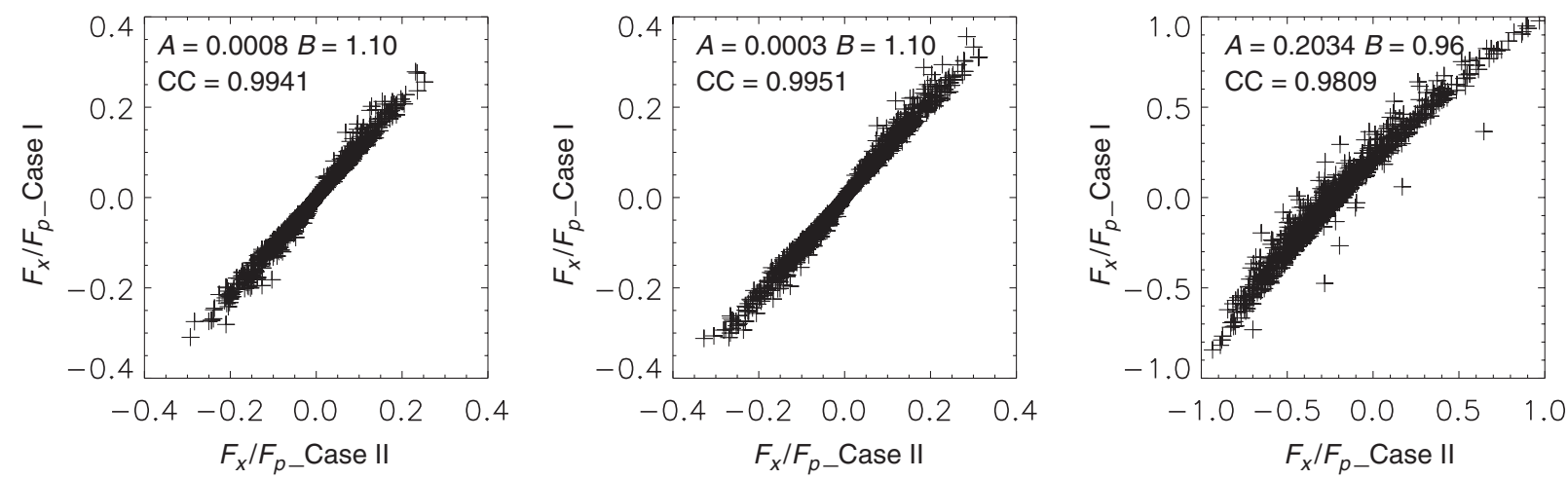

Figure 5. Scatter diagrams of $F_{x} / F_{p}, F_{y} / F_{p}$, and $F_{z} / F_{p}$ deduced from cases I and II, respectively, for the selected magnetograms.

strength. The results are consistent with those of Case I, only the amplitudes of $F_{z} / F_{p}$ decrease as $B_{z}$ increases.

To see the difference in results of $F_{x} / F_{p}, F_{y} / F_{p}$, and $F_{z} / F_{p}$ between two cases, the scatterplots of $F_{x} / F_{p}, F_{y} / F_{p}$, and $F_{z} / F_{p}$ of Case I versus the corresponding ones from Case II are shown in Figure 5. The correlations are calculated, which are $0.994,0.995$, and 0.980 for $F_{x} / F_{p}, F_{y} / F_{p}$, and $F_{z} / F_{p}$, respectively. Also, the linear fits $(y=A x+B)$ are done based on these scatterplots, the values of $A$ are $0.0008,0.0003$, and 0.2034 and of $B=1.10,1.10$, and 0.95 for $F_{x} / F_{p}, F_{y} / F_{p}$, and $F_{z} / F_{p}$, respectively. The correlation shows that there exists high consistency between these two cases. Nevertheless, the correlation of $F_{z} / F_{p}$ between two cases is not as good as those of $F_{x} / F_{p}$ and $F_{y} / F_{p}$. This may imply that more attention should be focused on the amplitude of $F_{z} / F_{p}$ in order to understand the extent of force-free.

\section{DISCUSSIONS AND CONCLUSIONS}

It is worth studying the force-free extent of the photospheric magnetic field together with the case of magnetic field extrapolation, since it has been assumed that the coronal magnetic field is force-free and the photospheric magnetic field should be matched observationally. In this paper, results of the force-free extent of the photospheric magnetic field are given by conducting statistical research, using 925 magnetograms corresponding to 925 active regions observed by the SMFT at HSOS.

A part of efforts to avoid the uncertainty of the data calibration is the employment of two sets of calibration coefficients to describe the force-free extent of the photospheric magnetic field. For Case I, the calibration coefficients $C_{\mathrm{L}}$ and $C_{\mathrm{T}}$ are 8381 and $6790 \mathrm{G}$, the mean values of absolute $F_{x} / F_{p}$, $F_{y} / F_{p}$, and $F_{z} / F_{p}$ for all selected magnetograms are 0.077 , 0.109 , and 0.302 , respectively; and for Case II, $C_{\mathrm{L}}$ and $C_{\mathrm{T}}$ are 10000 and $9730 \mathrm{G}$, the mean values of absolute $F_{x} / F_{p}$, $F_{y} / F_{p}$, and $F_{z} / F_{p}$ are $0.078,0.111$, and 0.251 , respectively. There are $17 \%$ and $25 \%$ magnetograms with a value of $F_{z} / F_{p}$ less than 0.1 for cases I and II, respectively; in other words, $17 \%$ of Case I and $25 \%$ of Case II are force-free. Although there are differences between the two cases, the correlation is as high as $0.994,0.995$, and 0.980 for $F_{x} / F_{p}, F_{y} / F_{p}$, and $F_{z} / F_{p}$, respectively. Consequently, we concluded that a large part of the photospheric magnetic fields does not belong to a force-free field. Therefore, before extrapolating a magnetic field, the force-free extent of the photospheric magnetic field should be adequately considered. We note that $F_{z} / F_{p}$ decreases with the increase of $B_{z}$, which is a more important parameter indicating whether a magnetic field is force-free or not, since the amplitude of $F_{z} / F_{p}$ is larger than those of $F_{x} / F_{p}$ and $F_{y} / F_{p}$. From Equation (10), it can be seen that $F_{z} / F_{p}$ may be neglected when the amplitude of $B_{z}$ is comparable to $\sqrt{B_{x}^{2}+B_{y}^{2}}$, while they are apparently different between $B_{z}$ and $\sqrt{B_{x}^{2}+B_{y}^{2}}$, then the amplitude of $F_{z} / F_{p}$ should be enlarged. According to Equations (8)-(10) and observatories as well, the correlations between $F_{x} / F_{p}, F_{y} / F_{p}$, and the magnetic field component are not evident.

The strengths of active region magnetic fields observed by the SMFT at HSOS are determined through calibration, under the conditions of a weak-field approximation and linear relations between the magnetic field and the Stokes parameters $I, Q, U$, and $V$. In addition, comparing the recent data obtained from a space satellite, the data of the SMFT at HSOS have lower resolution and more uncertainty of magnetic amplitudes. These disadvantages may affect the statistical results we have acquired. However, the statistical analysis associated with its results may have significance as a practical reference, because the SMFT has observed photospheric magnetic fields more than one solar cycles at HSOS and its reliability observations have been studied strictly and adequately. It is hoped that statistical results can be obtained on the basis of high-resolution data in the future.

\section{ACKNOWLEDGMENTS}

The authors thank the anonymous referee for helpful comments and suggestions. This work was partly supported by the National Natural Science Foundation of China (Grant Nos. 11203036, 10611120338, 10673016, 10733020, 10778723, 11003025, 11103037, 10878016, and 11178016), the National Basic Research Program of China (Grant No. 2011CB8114001), the Important Directional Projects of 
Chinese Academy of Sciences (Grant No. KLCX2-YW-T04), the Young Researcher Grant of National Astronomical Observations, Chinese Academy of Sciences, and the Key Laboratory of Solar Activity National Astronomical Observations, Chinese Academy of Sciences.

\section{REFERENCES}

Ai, G., \& Hu, Y. 1986, AcASn, 27, 173

Ai, G., Li, W., \& Zhang, H. Q. 1982, AcASn, 23, 39

Aly, J. J. 1984, ApJ, 283, 349

Aly, J. J. 1989, SoPh 120, 19

Amari, T., Aly, J. J., Luciani, J. F., Boulmezaoud, T. Z., \& Mikic, Z. 1997, SoPh, 174, 129

Bao, S. D., Pevtsov, A. A., Wang, T. J., \& Zhang, H. Q. 2000, SoPh, 195, 75

Bond, H. E., Mullan, D. J., O'Brien, M. S., \& Sion, E. M. 2001, ApJ, 560, 919

Chandrasekhar, S. 1961, Hydrodynamic and Hydromagnetic Stability (Chapter 13) (Oxford: Clarendon)

Demoulin, P., Bagala, L. G., Mandrini, C. H., Henoux, J. C., \& Rovira, G. 1997, A\&A, 256, 305

Gao, Y., Su, J. T., Xu, H. Q., \& Zhang, H. Q. 2008, MNRAS, 386, 1959

Gary, G. A., \& Hagyard, M. J. 1990, SoPh, 126, 21

Gary, D. E., \& Hurford, G. J. 1994, ApJ, 420, 903

Harvey, J. W. 1977, in Highlights of Astronomy, Vol. 4, ed. E. MULLER (Magnetic: D. Reidel Publishing Company), 223

He, H., \& Wang, H. 2008, JGR, 113, A05S90

Jefferies, J., Lites, B. W., \& Skumanich, A. 1989, ApJ, 343, 920

Jefferies, J., \& Mickey, D. L. 1991, ApJ, 372, 694

Krall, K. R., Smith, J. B., Jr., Hagyard, M. J., West, E. A., \& Cummings, N. P. 1982, SoPh, 79, 59

Lin, J., Ko, Y.-K., Sui, L., Raymond, J. C., Stenborg, G. A., Jiang, Y., Zhao, S., \& Mancuso, S. 2005, ApJ, 622, 1251

Liu, S., Zhang, H. Q., \& Su, J. T. 2011a, SoPh, 270, 89

Liu, S., Zhang, H. Q., Su, J. T., \& Song, M. T. 2011b, SoPh, 269, 41

Liu, H., Kuhn, J. R., \& Coulter, R. 2004, ApJ, 613, L177
Low, B. C. 1985, in Measurements of Solar Vector Magnetic Fields, Vol. 2374, ed. M. J. Hagyard (Washington, DC: NASA), 49

Metcalf, T. R., Jiao, L., McClymont, A. N., Canfield, R. C., \& Uitenbroek, H. 1995, ApJ, 439, 474

Metcalf, T. R., et al. 2006, SoPh, 237, 267

Mikic, Z., \& McClymont, A. N. 1994, in ASP Conf. Ser. 68, Solar Active Region Evolution: Comparing Models with Observations, ed. K. S. Balasubramaniam and G. W. Simon (San Francisco, CA: ASP), 225

Molodensky, M. M. 1974, SoPh, 39, 393

Moon, Y., Choe, G. S., Yun, H. S., Park, Y. D., \& Mickey, D. L. 2002, ApJ, 568, 422

Nindos, A., Patsourakos, S., \& Wiegelmann, T. 2012, ApJ, 748, L6

Priest, E. R., \& Forbes, T. G. 2002, A\&ARv, 10, 313

Sakurai, T. 1981, SoPh, 69, 343

Shibata, K., Masuda, S., Shimojo, M., Hara, H., Yokoyama, T., Tsuneta, S., Kosugi, T., \& Ogawara, Y. 1995, ApJ, 451, L83

Song, M. T., Fang, C., Tang, Y. H., Wu, S. T., \& Zhang, Y. A. 2006, ApJ, 649, 1084

Stenflo, J. O. 1973, SoPh, 32, 41

Su, J. T., \& Zhang, H. Q. 2004, ChJAA, 4, 365

Tiwari, S. K. 2012, ApJ, 744, 65

Tsuneta, S. 1996, ApJ, 456, 840

Wang, H. 1997, SoPh, 174, 265

Wang, T. J., Ai, G. X., \& Deng, Y. Y. 1996, AR, 23, 31

Wang, H., Ewell, M. W., Jr., Zirin, H., \& Ai, G. X. 1994, ApJ, 424, 436

Wang, J. X., Shi, Z. X., Wang, H. N., \& Lu, Y. P. 1996, ApJ, 456, 861

Wang, H., Yan, Y., \& Sakurai, T. 2001, SoPh, 201, 323

Wang, T., Zhang, H., \& Xu, A. 1994, SoPh, 155, 99

Wheatland, M. S., Sturrock, P. A., \& Roumeliotis, G. 2000, ApJ, 540,1150

Wiegelmann, T. 2004, SoPh, 219, 87

Wiegelmann, T., Inhester, B., \& Sakurai, T. 2006, SoPh, 233, 215

Wu, S. T., Sun, M. T., Chang, H. M., Hagyard, M. J., \& Gary, G. A. 1990, ApJ, 362, 698

Yan, Y., \& Sakurai, T. 2000, SoPh, 195, 89

Zhang, H. Q. 2000, SoPh, 197, 235 